\title{
Local Ultrasonic Resonance Spectroscopy: A Demonstration on Plate Inspection
}

\author{
J. Rus ${ }^{1}(1) \cdot$ C. U. Grosse ${ }^{1}$
}

Received: 22 October 2019 / Accepted: 10 March 2020 / Published online: 19 March 2020

(c) The Author(s) 2020

\begin{abstract}
Local ultrasonic resonance spectroscopy (LURS) is a new approach to ultrasound signal analysis, which was necessitated by a novel inspection method capable of the contact-free, localized, broadband generation and detection of ultrasound. By performing a LURS scan, it is possible to detect local mechanical resonances of various features and of the specimen itself. They are highly sensitive to local mechanical properties. By observing different parameters in the frequency spectrum (e.g., resonance amplitude and resonance peak frequency), geometrical, material and condition properties can be visualized for all of the scanning positions. We demonstrate LURS for inspection of a carbon fiber reinforced polymer plate. Local defect resonances of delaminations and a flat-bottom hole were detected in the frequency range from 25 to $110 \mathrm{kHz}$. Analyzing the higher frequency range $(0.3 \mathrm{MHz}$ to $1.5 \mathrm{MHz})$ of the same scan, the shift of the thickness resonance frequency of the plate and its higher-order resonance frequencies carry the information about the aluminum inclusions. LURS shows an advantage in characterizing the localized features of the specimens via contact-free ultrasonic inspection.
\end{abstract}

Keywords Local ultrasonic resonance spectroscopy $\cdot$ Thickness resonance $\cdot$ Local resonance $\cdot$ Ultrasonic inspection

\section{Introduction}

There are two fundamental approaches to describe the elastodynamic state of a stationary body. On the one hand, motion of the whole body (part) can be considered by observing its non-periodic or (partially) periodic deformations, i.e., theory of vibration or modal analysis. On the other hand, we can study how the mechanical waves distribute in the body, i.e., wave motion theory. The wavelength of the motion can be defined as a decisive criterium. It can be determined from the periodicity of the motion, by knowing the material properties (or wave velocities) of the body. If the wavelength is in an order of magnitude of the size of the body (part) or longer, it is reasonable to use the theory of vibration; if the wavelength is significantly smaller from the size of the body (part), the motion can be better described by wave theory.

However, a certain duality is still present for the transition between these two criteria, and it is especially pronounced

$\triangle$ J. Rus

janez.rus@tum.de

1 Chair of Non-Destructive Testing, Centre for Building Materials, Technical University of Munich,

Franz-Langinger-Straße 10, 81245 Munich, Germany for the motion of a broadband frequency. The experimental equipment used in our experiment allowed us to consider frequencies ranging from several $\mathrm{kHz}$ to the $\mathrm{MHz}$ range. A pressure wave velocity of $2700 \mathrm{~m} / \mathrm{s}$ for the test specimen used in this study yielded wavelengths from the meter to the sub-millimeter range. The mechanical behavior in the $4.1 \mathrm{~mm}$ thick specimen plate thus encompasses both regimes. Down to $25 \mathrm{kHz}$, the natural oscillation of a feature is excited together with up to $3-\mathrm{MHz}$ US waves, which have fully distinct thickness reflections. According to the described duality, the thickness reflections can also be interpreted as symmetric oscillations of the plate.

Resonant ultrasound spectroscopy (RUS) is an established method, in which the entire specimen (normally a cube) is excited with the ultrasound (US) in a broadband frequency range [1-3]. The integral material parameters of the specimen are obtained from the natural oscillation frequencies. Local ultrasonic resonance spectroscopy (LURS) uses the same principle, but applied locally.

In LURS, the specimen is excited locally, and its mechanical response is captured at a position nearby. Local resonances of US are acquired from the frequency spectrum of the captured signal. While performing a scan, it is possible to obtain material, geometrical, and other mechanical proper- 
ties for each of the scanning-grid locations, or to detect their spatial change.

The term 'local' is relative to the object size, and the requisite criterium is having a size significantly larger than the inspected region. The goal of LURS is to excite only a localized region of the object (e.g., a feature or defect) to capture the natural mechanical behavior thereof. The local region of the object responds with its local resonances. For example, in the case of plates, we can detect the local thickness resonance (TR).

It is evident that even locally excited motion will spread through the entire specimen. However, high frequency components of motion are more strongly attenuated as the distance from the excitation point increases. Thus, for the higher frequencies of the motion considered, we can localize the region of interest, and thereby still use the theory of vibration to approach the problem.

LURS extends local acoustic resonance spectroscopy (LARS) to higher frequencies. In the latter method, the specimen is locally excited by mechanical impact [4-7]. The mechanical response is captured in the air in the audible frequency range by a microphone. Local changes in the material properties affect the amplitude of characteristic frequencies, which can be plotted for all the scanning positions.

LURS uses US excited and captured in broadband frequency range without physically contacting the specimen in order to achieve free local vibrations of the specimen. At the same time, this approach allows us to perform a scan having repeatable inspection properties for all of the scanning locations. A recently developed system comprising a photoacoustic US excitation and broadband optical microphone made this possible.

In comparison to air-coupled ultrasound (ACU) inspection methods, in which, due to the coupling problem, only narrowband transducers are conventionally used, LURS opens a new dimension. Using light as an analogy, monochromatic US becomes colored. By exciting white US, the inspected object can be observed in US colors if the receiver is likewise sensitive to the full frequency spectrum.

To preserve all of the information, data captured by LURS need to be organized in a 3-dimensional matrix. We refer to it as a scan data matrix. This matrix has the following axes: scanning position $x$, scanning position $y$, and time. The amplitude value of the captured signal for all the time instances is registered for all the scanning grid positions. Furthermore, the captured signal is converted to the frequency domain individually for all the scanning positions. We then obtain a scan data matrix in the frequency domain with the following axes: scanning position $x$, scanning position $y$, and frequency. The amplitude value of the frequency components is registered for all of the scanning grid positions. The present research did not study the signal phase or time of arrival, which also contains information about the specimen. In LURS, the entire 3-dimensional scan data matrix in the time and frequency domains is analyzed. This is achieved by slicing the matrix into various cross-sectional images: $\mathrm{B}$-scans, $\mathrm{C}$-scans and Fscans [8]. The recently developed experimental equipment allowed us to acquire the broadband US spectrum at each grid point in the scanned area. Thus, the presentation of the data in frequency domain B-scans gained a great deal of importance. In the growing field of broadband US inspection, it is advantageous to refer to these as S-scans (spectrum scans).

In the following, we will provide a brief review of the previous experiments that analyzed broadband US spectrum for specimen characterization. These range from the 1970 s, when the first experiments were conducted using an immersion testing setup in which the broadband frequency range was more easily achieved through water coupling [9, 10], until the present. A review of the early applications of ultrasonic spectral analysis to nondestructive evaluation was provided by Fitting and Adler [11].

Ultrasonic spectroscopy is challenging when applied to ACU testing methods. However, there are some alternatives to piezoelectric transducers that have a frequency range broad enough for ultrasonic spectroscopy, e.g., electrostatic (or capacitance) transducers [12, 13]. By applying pulsed US excitation, the thickness resonance (TR) frequency of the specimen plates of different material or thickness was detected in a through-transmission setup using two electrostatic transducers.

Laser-induced ultrasound is an efficient alternative for generating broadband US since it allows generation of short shock waves by means of the thermal (an ablation regime is also feasible) expansion of the illuminated area directly in the specimen [14]. Spectral analysis of US captured via piezoelectric transducer [15, 16] and laser interferometer [17] was performed to obtain information about the specimen. In [15], the level of porosity in carbon fiber-reinforced polymer (CFRP) was measured by observing the resonance peak of attenuation (amplitude and bandwidth) in the frequency domain using water as a couplant medium. The porosity in a laser welding aluminum joint was detected in [17], by observing B-scans of different frequency-bandwidths. In [16] laser-generated US was captured by a piezoelectric transducer. The CFRP including flat-bottom hole and delamination was studied on C-scans and Lamb wave tomography images. A similar experimental setup as the one used in our experiment has already been applied to the inspection of impact damage in biocomposites [18]. The only parameter being analyzed on the US signal captured by the optical microphone was amplitude in the time domain.

An extensive study on local defect resonances (LDR) was conducted by Solodov and Kreutzbruck [19-21]. They analyzed the natural oscillations of flat-bottom holes, delaminations, undulations, and adhesive disbonds. The defects were excited by tunable contact and contact-free (piezoelec- 

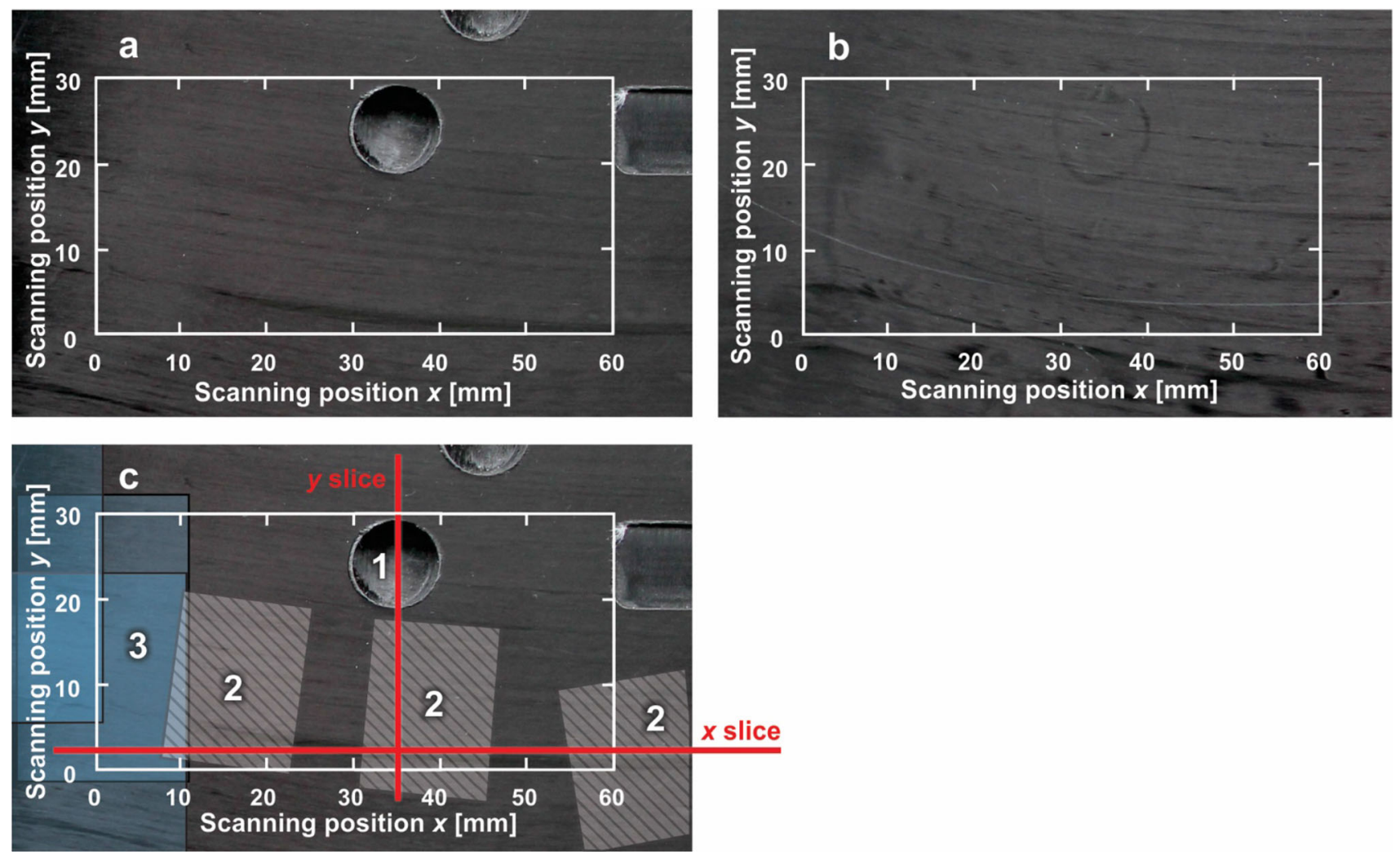

Fig. 1 The back (a) and the front (b) side of the specimen. In (c) the approximate locations of the flat-bottom hole (1), delaminations (2) and aluminum inclusions (3) are marked. The white rectangle with the coordinate system denotes the scan area

tric loudspeakers) transducers. For imaging of the defects, emitting sound at their local defect resonance, air-coupled transducers, microphone or laser vibrometers were used, depending on the frequency range of interest, which was mostly audible sound or near-US (several hundred $\mathrm{kHz}$ ). Their experiments were supplemented by separate experiments using a piezoelectric patch to activate and a laser vibrometer to detect the local defect resonances [22, 23].

In using LURS, we are combining the local defect resonance approach with the classical US inspection. The local resonance behavior of the specimen can then also be analyzed in the US frequency range, where the information-carrying frequencies of more delicate features (inclusions, material changes) are situated.

\section{Specimen Description}

The specimen was a $30 \times 30 \mathrm{~cm}$ CFRP (carbon fiber reinforced polymer) plate of $4.1 \mathrm{~mm}$ thickness [24]. The plate was made of 15 carbon fiber layers with a thickness of $0.27 \mathrm{~mm}$ $\left(0^{\circ}, 90^{\circ}\right.$ alternating orientation). Epoxy resin was used as a matrix. The scanned area of the specimen is marked with the white frame in Fig. 1. Red lines mark the cross-sectional locations in the $x$ and $y$ scanning directions in which detailed
LURS was performed in B-scans (Figs. 4 and 5) and S-scans (Figs. 6 and 7).

Three different features (simulated defects) were located in the inspected area of the specimen. The first was a flat-bottom hole (number 1) centered at $(35 \mathrm{~mm}, 25 \mathrm{~mm}$ ) (scanning position $x$, scanning position $y$ ). It had a diameter of $9.5 \mathrm{~mm}$. The remaining thickness under the hole was approximately $0.29 \mathrm{~mm}$. Due to the drilling process, the remaining layer was slightly bulged out of the surface, as seen in Fig. 1b).

The second feature type was a simulated delamination (number 2) created at three locations that lie (not fully) within the scan area. The each delamination was created by placing a pair of polytetrafluoroethylene (PTFE) films $(0.1 \mathrm{~mm}$ thickness) between the carbon fiber layers during the manufacturing process of the plate. The PTFE film pair had dimensions of approximately $15 \mathrm{~mm} \times 20 \mathrm{~mm}$. Delaminations were located at depths between 1.5 and $2 \mathrm{~mm}$ from the front side of the specimen. By embedding the PTFE pair in the plate, we achieved the mechanical detachment of the matrix between two fiber layers. The simulated delaminations are not clearly visible by computer tomography (CT). We assume that PTFE films are what we were actually detecting by $\mathrm{CT}$ rather than the delamination itself. 

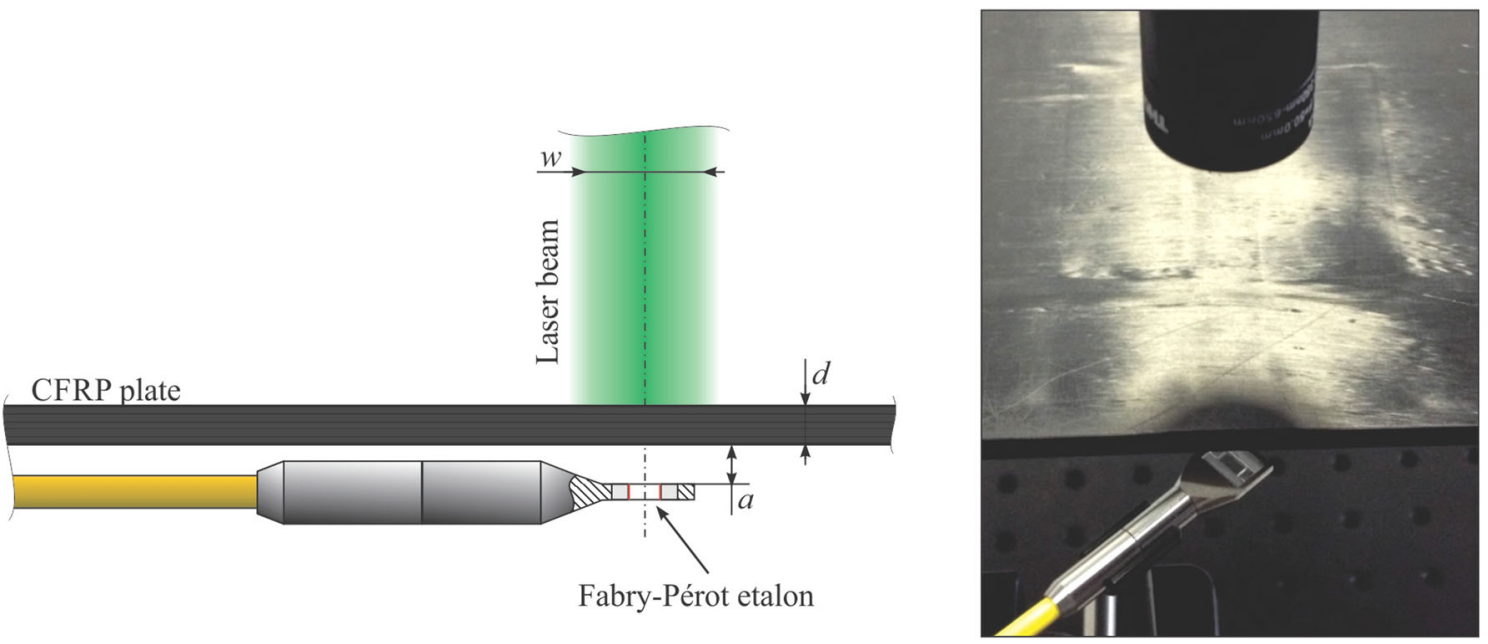

Fig. 2 Scheme and photo of the LURS experimental setup: US is excited by the pulse laser on the front side of the plate. The optical microphone is located on the other side of the plate

The third feature was located below $15 \mathrm{~mm}$ scanning position $x$ (number 3 ). In this area individual fiber layers were replaced by the three pieces of aluminum foil having approximately the same thickness. At least one carbon fiber layer lay between individual aluminum films. Consequently, the matrix came in contact with both sides of the film and the aluminum was adhesively connected to the CFRP, thus simulating the inclusion defect.

Due to the challenging (plate) geometry of the CT scan, the locations of the aluminum foils were determined at a limited precision. Their approximate location is marked with the blue overlapping squares in the area marked as (number 3 ) in Fig. 1. The foils were shifted with respect to one another in the in-plane direction. As a result, we had areas in the specimen in which either one, two, or three aluminum layers were above each other across the thickness of the plate. The number of aluminum layers can be identified in Fig. 1 by the blue color intensity.

\section{Description of LURS Setup}

Laser-induced US appears to be the only method capable of inducing high-intensity US over a frequency range broad enough to effectively perform LURS on CFRP plates several mm thick. We used a Nd-YAG frequency-doubled, qswitched pulse laser with a wavelength of $532 \mathrm{~nm}$ to generate the blast US waves in the specimen. The laser source was part of US excitation system provided by Xarion Laser Acoustics. The maximal laser pulse repetition rate was $20 \mathrm{~Hz}$. Over the output optic, the laser light was collimated on $8 \mathrm{~mm}$ diameter $w$ (95\% intensity level). The laser pulse peak intensity was low enough not to exceed the ablation threshold; the US was induced in the thermoelastic regime.
In LURS, a broadband US source needs to be combined with a broadband US detector. We used an Eta450 Ultra optical microphone made by Xarion Laser Acoustics. The optical microphone does not have any moving parts, as is normally the case for conventional US receivers (piezoelectric material or membrane). It measures the US directly in the air by measuring the change of the refractive index as acoustic waves travel through the Fabry-Pérot etalon [18, 25-27]. However, the housing of the microphone itself (or the etalon mirrors) can have a certain natural behavior which could affect the signal. The optical microphone has a frequency range from near-DC to approximately $3 \mathrm{MHz}$. The sensitivity is $100 \mathrm{mV} / \mathrm{Pa}$, and the self-noise at the full bandwidth (as specified by the manufacturer) is $10 \mathrm{mPa}$.

The US source and the detector were arranged in a through-transmission setup (Fig. 2) with the laser illuminating the front side of the plate as defined in Fig. 2. The optical microphone was placed in the middle of the laser beam axis on the other side of the plate. US was induced directly in the specimen by the laser pulse. On the receiver side, US was transmitted through an air gap of approximately $4 \mathrm{~mm}$ between the specimen and the sensitive part (Fabry-Pérot etalon) of the optical microphone. For LURS, it is advantageous to have the specimen mechanically released at the measurement location (ACU) in order to ensure free local mechanical oscillations. Since we are only considering local oscillations, the clamping of the whole specimen does not have a significant influence on the LURS results.

All of the results of the LURS analysis described in this work were obtained from a single scan 38 min long. The laser beam was used simultaneously with the optical microphone driven over the $60 \times 30 \mathrm{~mm}$ area of the CFRP plate while inducing the US at $301 \times 151$ grid points $(0.2 \mathrm{~mm}$ increment length). At each scanning location, the optical 

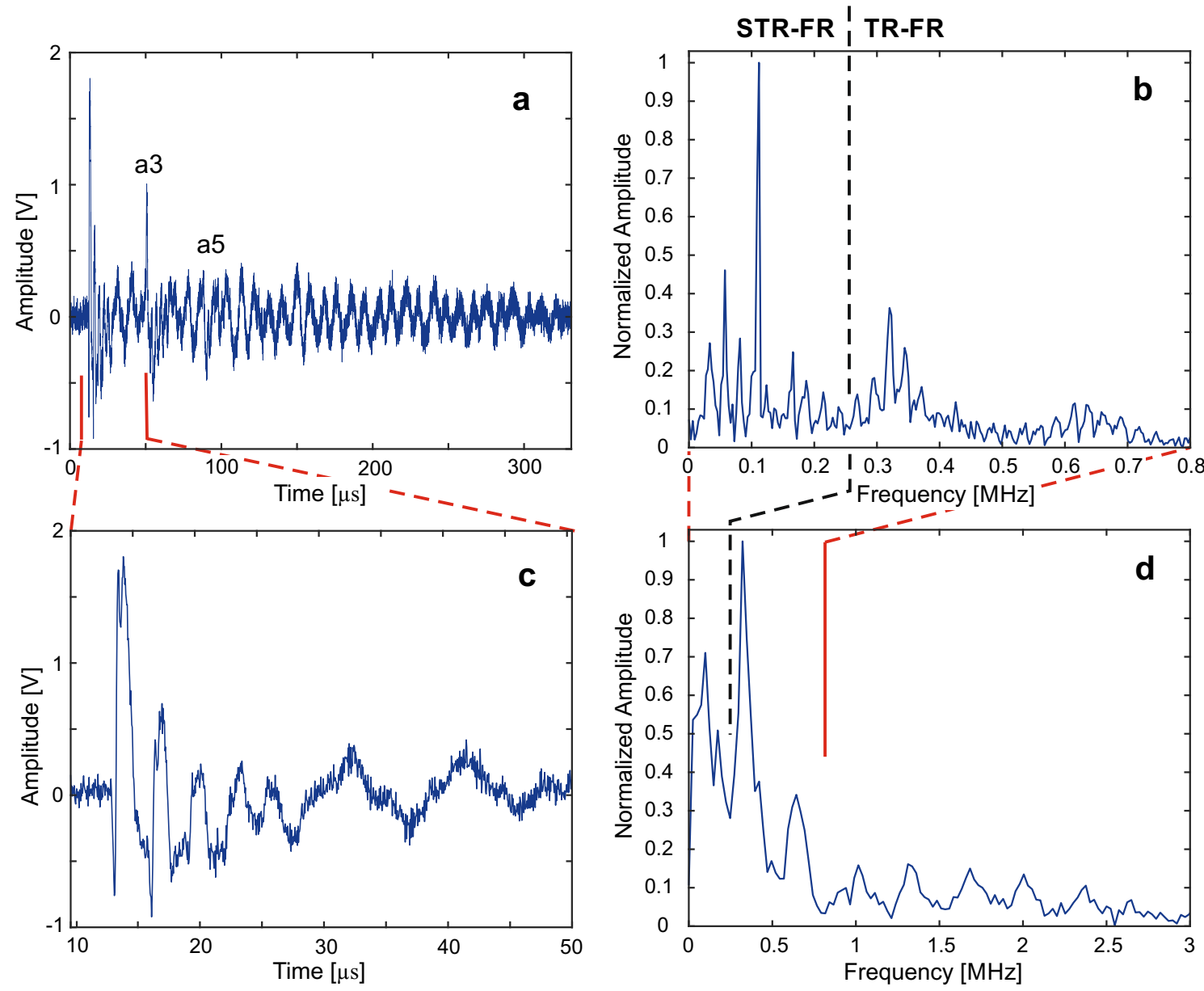

Fig. 3 The typical signal from optical microphone at the undamaged location with its frequency spectrum: in full length (long time interval) (a) and zoomed to the short time interval (c). b is the frequency spectrum of the long time interval signal and $\mathbf{d}$ is the frequency spectrum of the short time interval

microphone captured the US while being externally triggered by the control unit. No averaging was applied. The signal was digitalized (14-bit values) at a $25 \mathrm{MHz}$ sampling frequency. The sufficient spatial resolution was achieved by small-sized sensitive area of the optical microphone: approximately $0.4 \mathrm{~mm}^{2}$, as specified by the manufacturer. For additional details about the experimental setup, please refer to [28].

\section{Results of LURS Scan and Discussion}

\subsection{Typical Signal at Undamaged Location}

Since the present research is studying the mechanical behavior of plates over a broadband frequency range, it is advantageous to define two ranges of interest in the frequency domain. We refer to them as the sub-thickness resonance frequency range (STR-FR) and the thickness resonance frequency range (TR-FR). In the STR-FR, local defect reso- nances, the lower-mode asymmetric plate oscillations (lamb waves), and mechanical oscillation of the full body predominate. The TR-FR was comprised of the TR (first symmetric plate oscillation-lamb wave) and its high-order resonances. The dividing frequency was defined as the TR frequency reduced by a certain factor (e.g., 0.8 ). As a result, we ensured that all of the observed phenomena of the TR lay in the TR-FR. The dividing frequency was dependent on the US pressure wave velocity (perpendicular to the fiber orientation) and the plate thickness. For our specimen, this amounted to $0.8 \times 330 \mathrm{kHz}=264 \mathrm{kHz}$ since the TR frequency of the specimen was approximately $330 \mathrm{kHz}$. We require a longer time window for the signal in the STR-FR signal analysis. In contrast, a shorter time window is sufficient for the TR-FR. We are defining a short time window as the interval confined between the signal's first brake moment and the first in-air reflection of US between the optical microphone and the specimen (marked as a 3 in Fig. 3). Long time window is the time interval of the full wave train. This separation is necessary because the low frequency oscillation lasts longer 

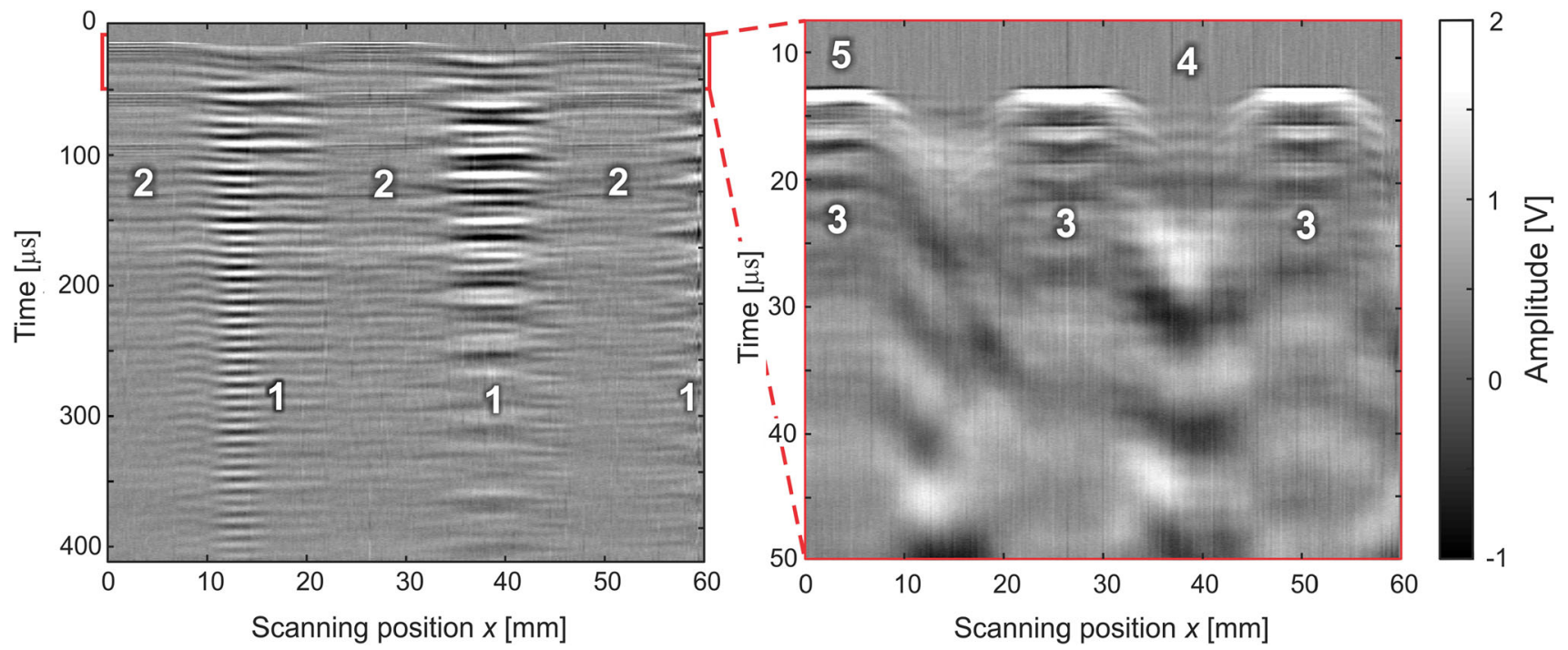

Fig. 4 B-scan at $y=3 \mathrm{~mm}$ scanning position. The cross-sectional cut goes over three delaminations (located at number 1) and inclusion (located at number 5)

than the time between the in-air US reflection between the optical microphone and the specimen. In another respect, these reflections disturbed the high-frequency analysis of the signal. The following analysis provides a separate indication of which frequency range was used.

Figure 3a shows the full length of the signal (long time interval) along with its frequency spectrum Fig. 3b. The red lines mark the short time interval, an exploded view of which is shown in Fig. 3c. Figure 3d shows a frequency spectrum of a short time interval signal. The signal shown refers to the scanning position at which the laser illuminates the undamaged part of the specimen. The amplitudes of the frequency spectrum are normalized to its maximum value.

In Fig. 3a, one can observe at least two in-air reflections of US between the specimen and the optical microphone (a3 and a5). They can be distinguished as higher-frequency signal oscillations superimposing the lowerfrequency signal oscillations, which result from the bending-mode oscillations of the specimen plate or the LDR oscillations. The latter can be detected also at the damage-free area, since they have higher amplitude and are less attenuated in air than the higher frequency US components (e.g. TR). This effect can also be observed in the Fig. 6.

In zooming in to the short time interval, we can observe in-plate wave reflections at a period of approximately $3.0 \mu \mathrm{s}$. Given this value and the plate thickness, we can calculate the approximate (and averaged over the plate thickness) pressure wave US velocity in CFRP perpendicular to fiber direction: $2 \times 4.1 \mathrm{~mm} / 3.0 \mu \mathrm{s}=2700 \mathrm{~m} / \mathrm{s}$. The same velocity can also be obtained by the TR of the plate: $330 \mathrm{kHz}$. This is in agreement with the value expected for CFRP.
The shape of the frequency spectrum changes by reducing the length of the signal. Numerical frequency resolution decreases-being inverse value of the time window. Lower frequencies become less pronounced. Their amplitudes decrease while the frequency peaks in the high-frequency range have a more defined shape (comparing Fig. 3b-d). The TR and seven higher-order resonances of TR are visible.

\subsection{B-scans}

In the following, the data obtained by a single LURS scan are presented in B-scans, C-scans, F-scans and newly defined $\mathrm{S}$-scan (B-scans in frequency domain). Figure 4 shows a characteristic cross-sectional image at $y=3 \mathrm{~mm}$ scanning position. The abscissa is scanning position $x$, and the ordinate is time. The signal amplitude is shown in grayscale, as captured by optical microphone in a B-scan.

The cross-sectional cut is performed over all three delaminations and the inclusion area (aluminum foils) (refer to Fig. 1c).The B-scan is shown in the long and short time window.

In Fig. 4 (above and below number 1), we can observe lowfrequency, long-lasting natural oscillations of the delaminations, which have a relatively high amplitude. The recorded oscillation is a consequence of a complex mechanic behavior of a delamination. However, the shape of the signal for the compression and the extension phase of the delamination is the same, having the inverted sign-no amplitude hysteresis can be observed. Thus the presence of the non-linear mechanic oscillation of the delamination cannot be deduced from the recorded signal [29]. 


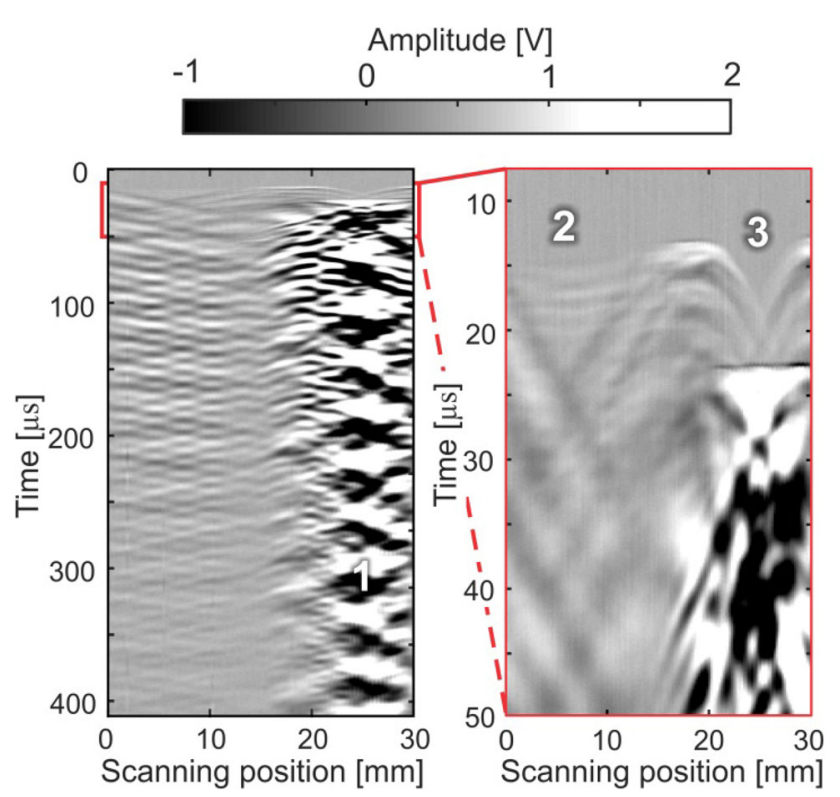

Fig. $5 \mathrm{~B}$-scan at $x=35 \mathrm{~mm}$ scanning position. The cross-sectional cut goes over a delamination (located at number 2) and flat-bottom hole (located at number 3)

We can see at least two in-air reflections of the US between optical microphone and specimen plate (Fig. 4: thin high frequency lines above number 2). If we zoom into the small time window (before in-air reflections), we can distinguish in-plate US reflections (Fig. 4: white lines above number 3). These reflections are present only in the non-delaminated areas of the specimen. Therefore, they are a good indicator of the delamination in which the US of this frequency is strongly dissipated. The oscillations with a frequency approximately double of the TR are present at the delaminated area, which are due to the reflections of US on the delaminations and characteristically arrive before the back-side plate echo (first in-plane reflection). They have comparatively small amplitude and are thus hardly seen in Fig. 4 below number 4 . The reflections from the inclusion defects cannot be significantly distinguished below number 5 in Fig. 4.

Figure 5 shows a B-scan at $x=35 \mathrm{~mm}$ scanning position. The cross-sectional cut is performed over the delamination and the flat-bottom hole (refer to Fig. 1c).

The remaining layer of the flat-bottom hole is strongly excited in its natural oscillations by the laser pulse. This can be observed in periodical changes of black and white color below and above number 1 , where the pattern of the natural oscillation can be observed. Below the number 2, we can observe the lack of high-frequency in-plate reflections that is caused by the delamination. The arrival of the first high-frequency wave train is delayed at the flat-bottom-hole location because the US needs to travel the longer distance through air there (below number 3), comparing to the rest of the scanning area.

\subsection{S-scans (B-scans in Frequency Domain)}

Additional information can be obtained from the signal via a spectral analysis thereof. We thus applied the Fourier transform to a time signal for all of the scanning positions. As a result, we obtained a second 3-dimensional data matrix with the following axes: scan direction $x$, scan direction $y$, and frequency. Each matrix element is a relative amplitude value (referring to the maximal of the whole matrix) of certain frequency and at certain scanning location.

Figure 6 shows a cross-sectional image of scan data matrix in the frequency domain at $y=3 \mathrm{~mm}$ scanning position. Figure 6 is Fig. 4 converted to the frequency domain. Again, we analyzed the signal in two frequency ranges: TR-FR (Fig. 4 left) and STR-FR (Fig. 4 right). For the TR-FR analysis of the signal, we used the small time window, and the long time window for the STR-FR analysis. Therefore, the zoomed Sscan (Fig. 4 right) has a better frequency resolution than the full range (Fig. 4 left). To make higher-order resonances of the TR more apparent, the amplitude shown in grayscale at the left of Fig. 4 left was expressed relatively to the maximum value of TR-FR and not to the maximal value of the full frequency range. The data presentation is therefore more transparent than when using logarithmic grayscale values.

The LDR of the delaminations are clearly visible (frequency peaks above number 1 in Fig. 6) at $33 \mathrm{kHz}, 58 \mathrm{kHz}$, $83 \mathrm{kHz}$, and $110 \mathrm{kHz}$. Different delaminations respond with different characteristic natural frequencies. The frequency spectrum can therefore be seen as the delamination's footprint. Further analysis of the delamination's LDR is performed in Fig. 8.

The TR is clearly visible at the delamination-free area (frequency peak at approximately $330 \mathrm{kHz}$ above number 2 and 3 in Fig. 6). We can also observe higher-order resonances of TR (above and below number 2 and 3 in Fig. 6). The US velocity in aluminum is higher than in CFRP (parallel to fiber orientation). Therefore, the in-plate reflections in the area of the specimen with inclusions have slightly shorter periods than reflections in the full-CFRP portion of the specimen. The increase of TR frequency in the inclusion area is hardly distinguishable (frequency peak at approximately $360 \mathrm{kHz}$ above number 3 in Fig. 6), however, it is significant enough to make the inclusions visible in an F-scan (Fig. 10a).

Figure 7 shows a cross-sectional image of the scan data matrix in frequency domain at $x=35 \mathrm{~mm}$ scanning position. Figure 7 is Fig. 5 converted to the frequency domain.

The flat-bottom hole in the specimen plate has a natural frequency at $25 \mathrm{kHz}$ (Fig. 7, number 1). The higher intensity of the US is transmitted through the remaining thickness of the flat-bottom hole as compared to the full-thickness plate.

The LDR of the delamination has lower amplitude comparing to the LDR of the flat-bottom hole. They are located at number 2, but not visible in the grayscale range of Fig. 7 . 

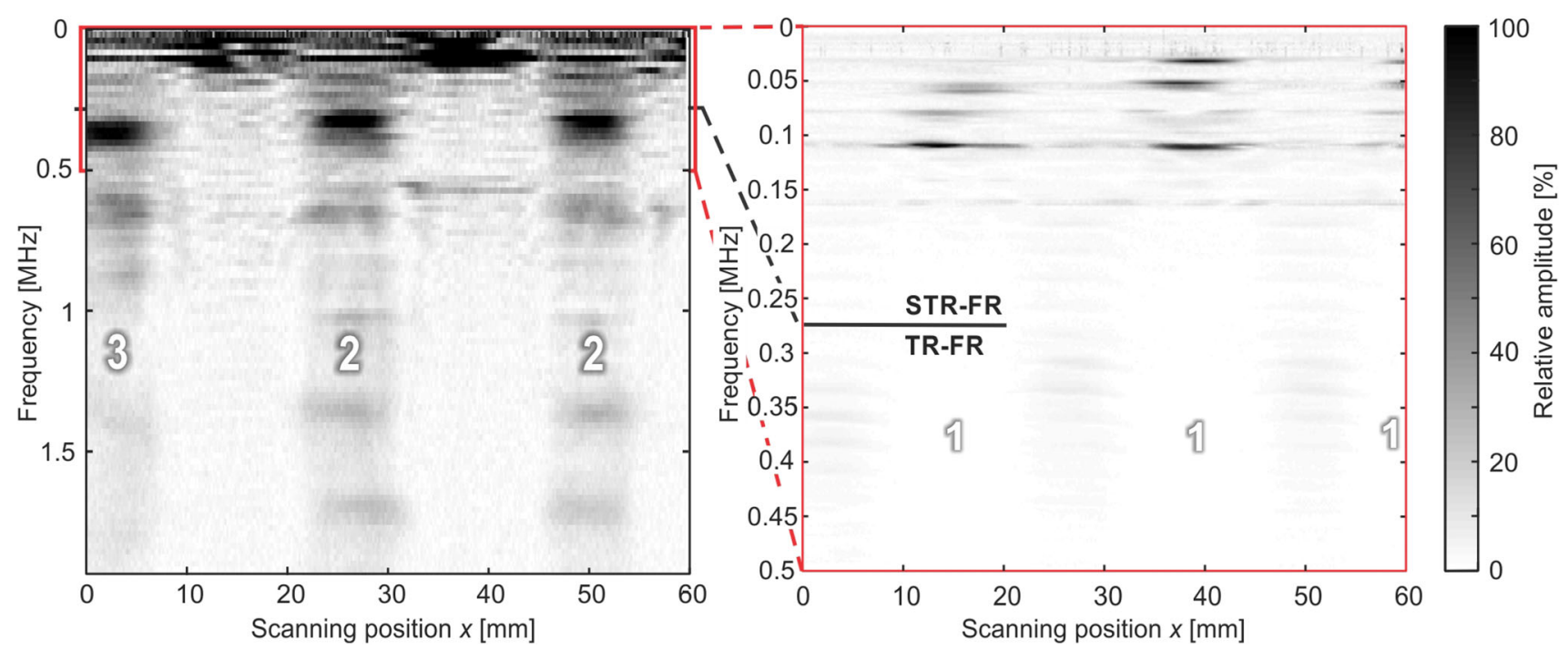

Fig. 6 S-scan (B-scan in frequency domain) at $y=3 \mathrm{~mm}$ scanning position. The cross-sectional cut goes over three delaminations (located at number 1) and inclusion (located at number 3)

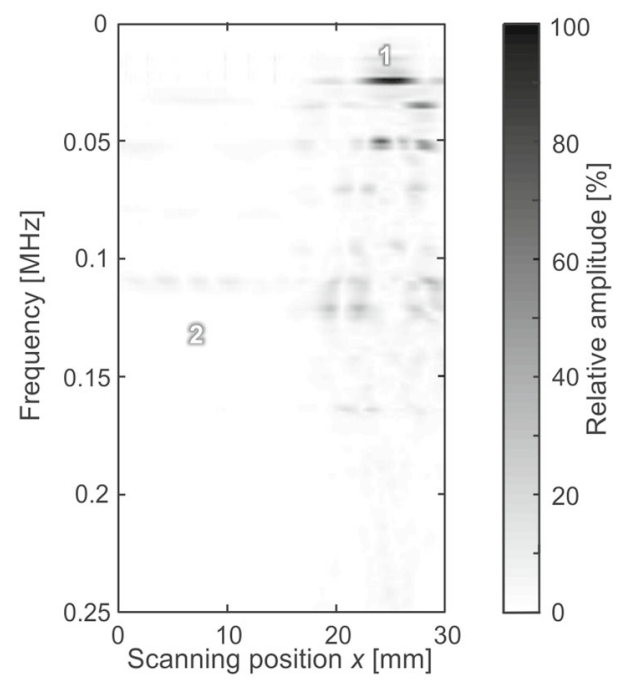

Fig. 7 S-scan (B-scan in frequency domain) at $x=35 \mathrm{~mm}$ scanning position. The cross-sectional cut goes over a delamination (located at number 2) and flat-bottom hole (located at number 1)

\subsection{C-scans}

We first analyzed the STR-FR which was of interest for the local resonance analysis of the features. Regarding C-scans, it must be specified how the amplitude value was obtained. Commonly, mean amplitude (or maximal amplitude) within the specified frequency interval or time window is coded in grayscale. If the frequency (or time) interval is comparatively small (e.g. below $5 \%$ of its mean value), specifying the mean value suffices.

Figure 8 shows $\mathrm{C}$-scans obtained from the frequency domain. In this case, the 3-dimensional scan data matrix in frequency domain is cut in the scanning position-scanning position plane (the remaining two orthogonal slice directions are S-scans in Figs. 6 and 7). For each C-scan, the frequency at which the cross-sectional image was obtained is explicitly labeled. Note that the amplitude value represents the average over the frequency discretization interval. The frequencies were chosen to fit the LDR frequency peaks of the flat-bottom hole and delaminations (frequency peaks above number 1 in Fig. 6 and below number 1 in Fig. 7). For all the scanning locations, the amplitude value is expressed relative to the maximal amplitude value of each respective $\mathrm{C}$-scan.

At $25 \mathrm{kHz}$, we can observe the flat-bottom hole oscillating at its $(0,1)$ eigenmode (Fig. 8, number 1$)$. The vibration of the delamination is a complex mechanical problem. It was researched on a numerical model in [29-31]. Some typical eigenmode shapes of the delamination are observable at their LDR frequencies in the Fig. 8. The two delaminations appear to oscillate at their $(1,1)$ eigenmode at different natural frequencies-33 kHz and $46 \mathrm{kHz}$ (Fig. 8, number 2 ). The frequencies at which the delaminations oscillate at higher modes are similar for both delaminations. At $58 \mathrm{kHz}$, the amplitude shape of LDR reminds on $(1,2)$ (number 3 ), at $83 \mathrm{kHz}$ on $(1,3)$ (number 4) and at $110 \mathrm{kHz}$ on $(1,4)$ eigenmode (number 5).

We estimated the order of magnitude of the LDR frequencies of the delaminations and the flat-bottom hole. In the first approximation, the oscillations of the flat-bottom hole and delaminations can be modeled by the analytical solution for circular and rectangular plate, respectively [20, 32]. The following assumptions were made for this simplified model: the deformability of the damage is much higher than that of the undamaged plate- the clamped edge is used 


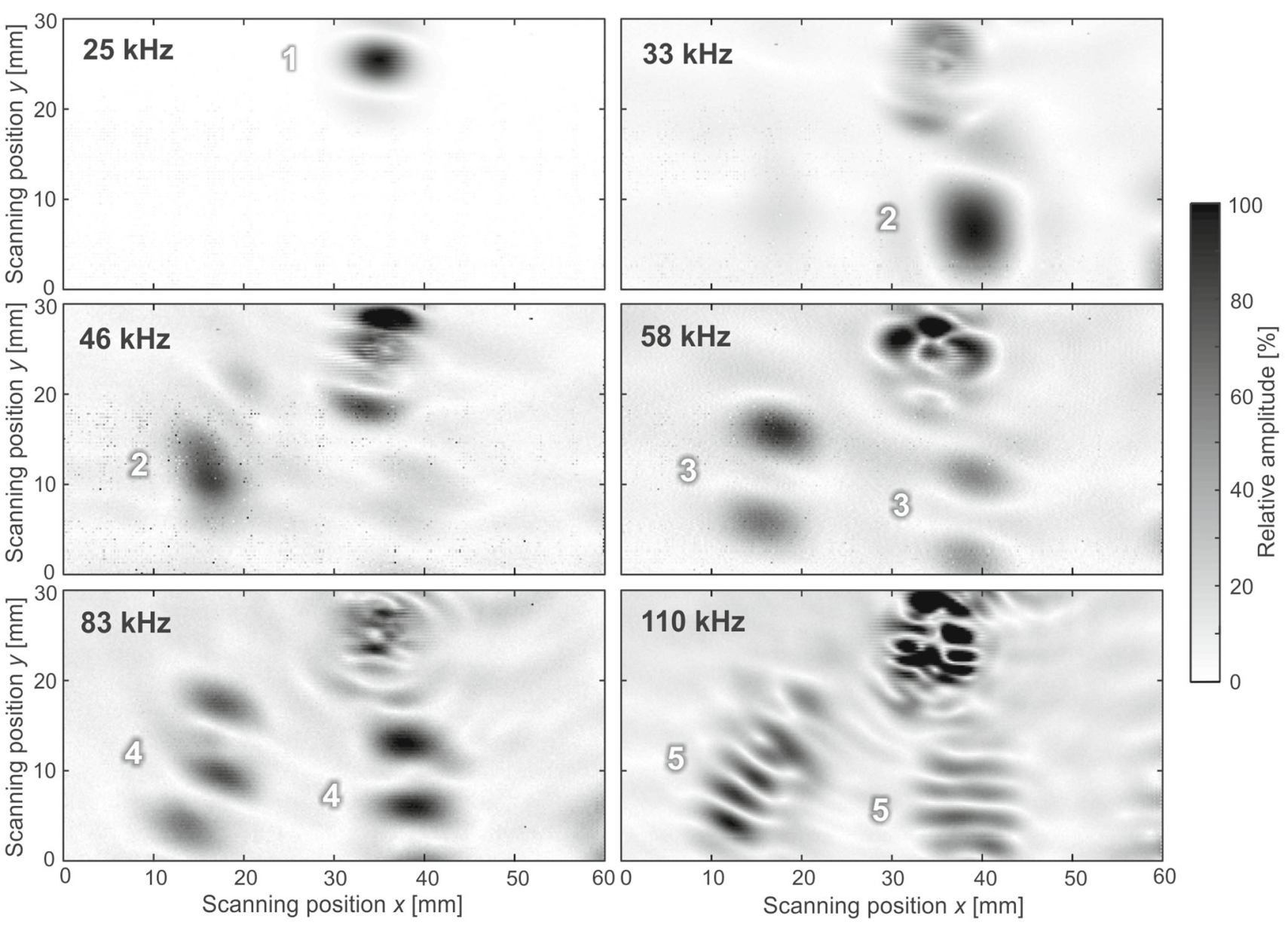

Fig. $8 \mathrm{C}$-scan at selected frequencies labeled in upper-left corner. Shapes that reminds on eigenmodes of flat-bottom hole (number 1) and delaminations (number $2-5$ ) are visible

as the boundary condition; the composite material is homogeneous and isotropic with a Young's modulus of $68 \mathrm{GPa}$ (mean value), Poisson's ratio of 0.3 , and mass density of $1600 \mathrm{~kg} / \mathrm{m}^{3}$ (values for the material used: T700/MTM57); for the delaminations, the interface of the disbanded laminates is mechanically decoupled and located in the middle of the plate thickness. Assuming these simplifications, the LDR frequencies can be calculated from the equations described in [32]. The first LDR frequency results in approximately $10 \mathrm{kHz}$ for the flat-bottom hole and approximately $80 \mathrm{kHz}$ for the delaminations.

The seventh C-scan is shown in Fig. 9, where the mean amplitude value of the US frequencies in TR-FR (between $264 \mathrm{kHz}$ and $3 \mathrm{MHz}$ ) is coded in the grayscale. The upper frequency limit ( $3 \mathrm{MHz}$ ) was defined according to the optical microphone's sensitivity range. The US amplitude is expressed relative to the maximal value of all the scanning position, excluding the flat-bottom hole. The US amplitude in TR-FR is significantly higher there, because of the small thickness of the remaining layer under the hole (number 1 in
Fig. 9). This can be observed also in S-scan in Fig. 7 below number 1 .

The TR-FR is highly sensitive with respect to delaminations. The TR amplitude peak, with its higher-order resonances, is strongly attenuated due to the detached fiber layers. Almost no US of this frequency range is transmitted through the delaminations (number 2 in Fig. 9). Furthermore, flat-bottom hole is clearly visible (number 1 in Fig. 9). Aluminum inclusions are not distinguishable.

\subsection{F-scans}

While the US amplitude in TR-FR is carrying the information about the attenuation level of US in specimen, it is the most suitable for the detection of features which deliver additional material-air interfaces (cracks, delaminations, kissing bonds, porosity, voids). In contrast, if the feature does not cause comprehensive impedance changes (i.e. order of magnitude), almost the same US intensity is transmitted through the feature as through the feature-free material. As a result, changes in mechanical properties of the material or inclusions 


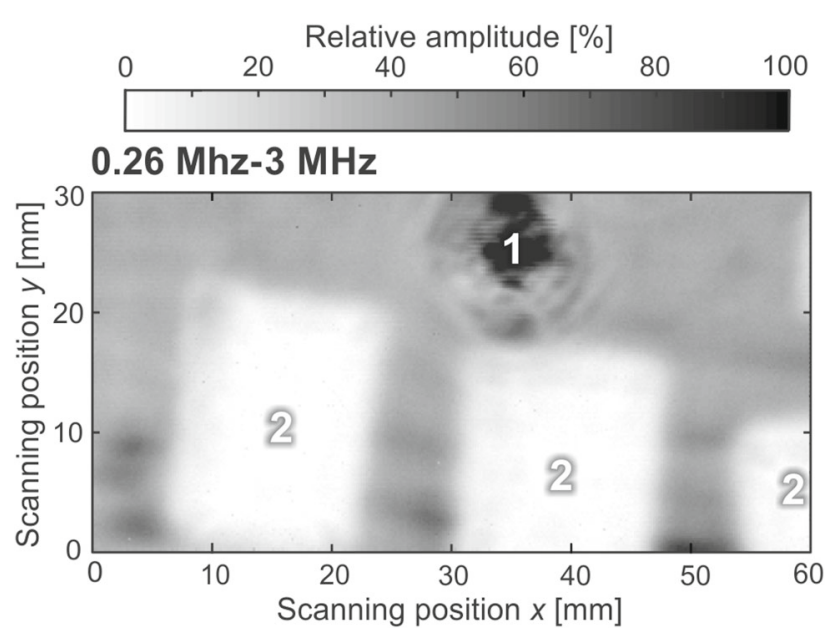

Fig. $9 \mathrm{C}$-scan with the mean amplitude value in the frequency interval $0.26-4 \mathrm{MHz}$ coded in grayscale. Flat-bottom hole (number 1) and delaminations (number 2 ) are clearly visible. Aluminum inclusions are not distinguishable

of foreign material (impurities) cannot be detected. However, they have a significant influence on the local resonance of the specimen - on the TR and its higher-order resonances in the case of plates. On the other hand, we can also interpret it according to the wave theory: depending on the local mechanical properties of the plate, the US velocity locally changes, which can be seen in the change of the period of the in-plate reflections.

The US velocity (longitudinal waves) in aluminum is approximately $6300 \mathrm{~m} / \mathrm{s}$, which is a factor 2.3 larger than the specimen plate used in this experiment (approximately $2700 \mathrm{~m} / \mathrm{s}$ perpendicularly to fiber orientation). For each of the CFRP layers (out of 15) which were exchanged by aluminum foil, the TR frequency increases by approximately $8.7 \%(1.3 / 15)$ from the initial TR frequency-i.e. $28.6 \mathrm{kHz}$ for our specimen.

In the F-scan shown in Fig. 10, the frequency peak value of the local TR of the specimen plate is coded in grayscale for all the scanning positions. To visualize the aluminum inclusions, the first TR (a) and fourth higher harmonic (b) turned out to be the most suitable. This choice depends on the nature of this feature and on the experimental setup itself (the optical microphone's frequency range and its frequency dependent sensitivity). The frequency peak of the first TR is determined in the range of $310-370 \mathrm{kHz}$, and the fourth higher harmonic in the range of 1.32-1.44 MHz. Grayscale is expressed absolutely in separate bars.

In contrast to the $\mathrm{C}$-scan, the $\mathrm{F}$-scans reveal also the aluminum inclusion (number 1). It is possible to determine the area where at least one inclusion is located, but not the exact number of overlapping foils. The first TR exhibits a significant decrease in frequency at scanning positions $y$ higher than $23 \mathrm{~mm}$, where aluminum inclusions are located (num-
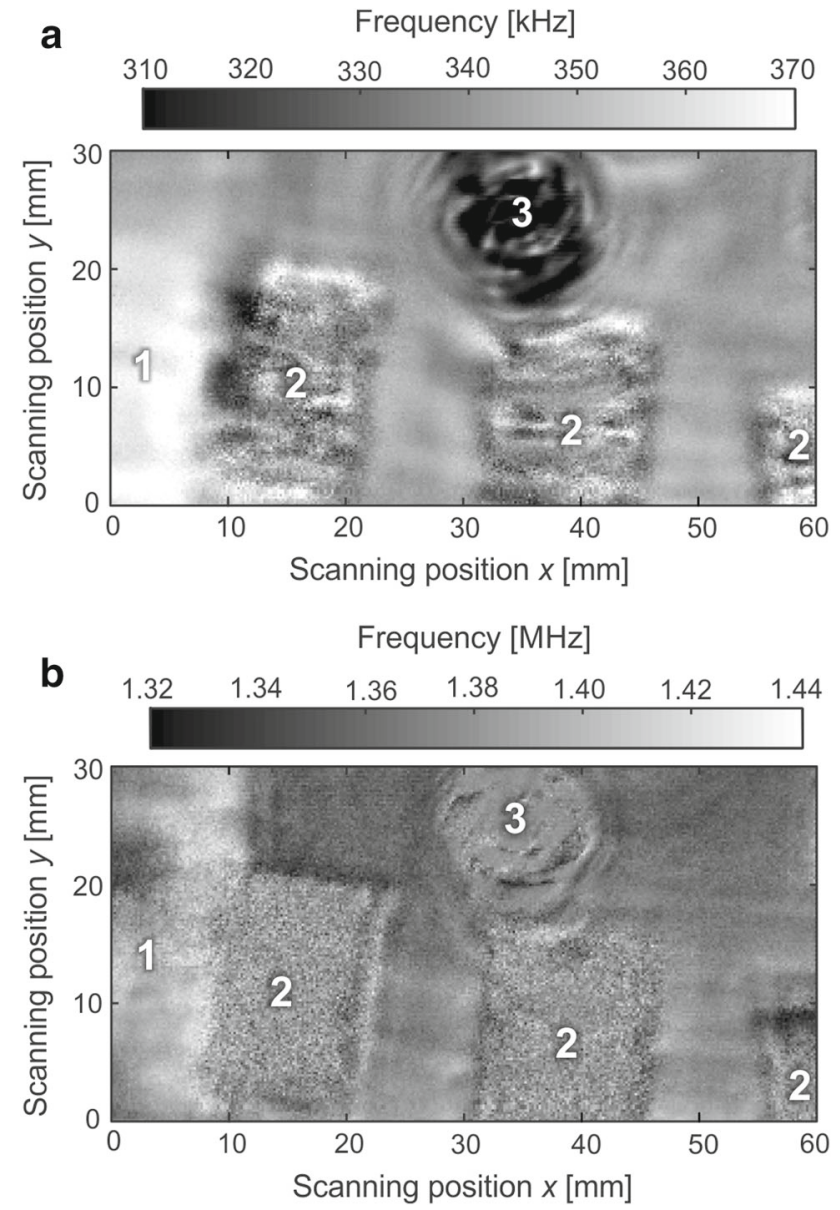

Fig. 10 F-scan of $0.31-0.37 \mathrm{MHz}$ (a) and $1.32-1.44 \mathrm{MHz}$ (b) frequency range. The frequency of first thickness resonance (a) and its forth higher harmonic (b) is coded in grayscale

ber 1 in Fig. 10a). The frequency transition correlates with the horizontal borderline of one inclusion. The second (vertical) borderline cannot be clearly detected from the first TR frequency. The surface, where at least one inclusion is located, can be more easily determined from forth higher harmonic of TR (Fig. 10b). The borderline is clear at the area with no delamination. In contrast, the intrusions cannot be detected, if they overlap with the delamination, because almost no US of TR-RF is transmitted through the specimen there. Therefore, the delaminations can be detected as the surfaces with higher noise level in Fig. 10 (number 2). Their boundaries can be determined comparatively well. Flat-bottom hole, which transmits high intensity of US in TR-FR range, is located at number 3 .

\section{Conclusions}

We introduced a new US inspection and signal interpretation approach, which was given the name of LURS. It was initi- 
ated by a novel experimental setup which is capable of the effective generation and detection of US ranging from several $\mathrm{kHz}$ to several $\mathrm{MHz}$. We considered the full frequency range integrally by way of observing the local resonance in US spectrum.

The LURS can be described as consisting of the following steps:

- broadband local excitation and acquisition of the US for all the scanning positions,

- organizing the data in 3-dimensional data matrix and its conversion to frequency domain,

- investigation of data matrix by observing B- and S-scans and identification of the characteristic frequencies, which carry the information about the features of the specimen. Characteristic frequencies can either be a local resonance of the features/defects (STR-FR for plates) or local resonance of the specimen itself (TR-FR for plates). A parameter for visualizing the features can also be an amount of high frequency US components-this is complementary to classical ultrasonic inspection.

- visualization of the features, by plotting amplitude or frequency shift of characteristic frequencies in C- and Fscans,

- classification of the features according to its form and local resonance frequency.

In this work, we demonstrated LURS on a CFRP plate that included a flat-bottom hole, simulated delaminations and aluminum inclusions. It is possible to visualize all of these features through LURS. We determined the LDR of the flatbottom hole and of simulated delaminations. We visualized the delaminations via the absence of high frequency components of US. We showed that aluminum inclusions effect TR and its fourth higher harmonic. This was visualized through the F-scans.

The advantages of LURS are the following. It makes the observation of local resonant behavior and ultrasonic wave propagation possible by performing a single measurement. The type and form of the feature can be classified. Since it is based on US, LURS is more highly sensitive to delaminations and other features that have a strong effect on mechanical properties of the specimen in comparison to radiography methods. Compared to classical US methods, LURS can be ad hoc more sensitive to certain features since it detects changes of local resonance behavior of the specimen which are strongly dependent on the local mechanical (geometrical, material etc.) properties. It is contact-free and allows relatively fast performance of a scan.

The disadvantages are that LURS - at this stage of development-requires manual analysis of the spectrum in order to determine characteristic information-carrying frequencies. Certain knowledge of the nature of single feature is required for this purpose. LURS necessitates handling with higher data quantities (scan data matrix). Since LURS is US-based, it is less sensitive to aluminum inclusions than radiology methods. However, this can be seen also as an advantage, since it allows selective detection of features that affect the mechanical properties of the specimen (e.g., adhesive disbonds between aluminum and CFRP). The disadvantages are further associated with laser safety.

The goal of our future work is to develop a computer-based tool which will be able to resolve the local specimen's properties from the US spectrum obtained by means of LURS. In terms of reverse engineering, geometrical, material and quality condition properties of the specimen are to be obtained automatically. The characteristic information-carrying frequencies of the US spectrum can also be obtained by means of machine learning algorithms.

Acknowledgements Open Access funding provided by Projekt DEAL. The authors acknowledge Dr.-Ing. Otto and Karla Likar foundation for the financial support, and XARION Laser Acoustics for providing the experimental equipment.

Open Access This article is licensed under a Creative Commons Attribution 4.0 International License, which permits use, sharing, adaptation, distribution and reproduction in any medium or format, as long as you give appropriate credit to the original author(s) and the source, provide a link to the Creative Commons licence, and indicate if changes were made. The images or other third party material in this article are included in the article's Creative Commons licence, unless indicated otherwise in a credit line to the material. If material is not included in the article's Creative Commons licence and your intended use is not permitted by statutory regulation or exceeds the permitted use, you will need to obtain permission directly from the copyright holder. To view a copy of this licence, visit http://creativecomm ons.org/licenses/by/4.0/.

\section{References}

1. Migliori, A., Sarrao, J.L.: Resonant Ultrasound Spectroscopy : Applications to physics, Materials Measurements, and Nondestructive Evaluation. Wiley, New York (1997)

2. Zadler, B.J., Le Rousseau, J.H.L., Scales, J.A., Smith, M.L.: Resonant Ultrasound Spectroscopy: theory and application. Geophys. J. Int. 156(1), 154-169 (2004). https://doi.org/10.1111/j.1365-246 X.2004.02093.X

3. Maynard, J.: Resonant ultrasound spectroscopy. Phys. Today 49(1), 26-31 (1996). https://doi.org/10.1063/1.881483

4. Jüngert, A., Grosse, C., Krüger, M.: Local acoustic resonance spectroscopy (LARS) for glass fiber-reinforced polymer applications. J Nondestruct Eval 33(1), 23-33 (2014). https://doi.org/10.1007/s1 0921-013-0199-3

5. Jatzlau, P., Müller, M., Grosse, C.U.: Identification of flawed CFRP samples using local acoustic resonance spectroscopy (LARS). In: WCNDT, 19th World Conference on Non-destructive Testing, 2016

6. Jatzlau, P., Grosse, C.U.: Local acoustic resonance spectroscopy: an escalation approach for fast non-destructive testing. In: 10th International Symposium on NDT in Aerospace (2018)

7. Grosse, C.U., Jüngert, A., Jatzlau, P.: Local Acoustic Resonance Spectroscopy, pp. 1-24. Handbook of Advanced Non-Destructive Evaluation. Springer, Berlin (2018) 
8. EN ISO 5577:2017. Non-destructive testing-Ultrasonic testing-Vocabulary

9. Brown, A.F.: Materials testing by ultrasonic spectroscopy. Ultrasonics 11(5), 202-210 (1973). https://doi.org/10.1016/0041-624 X(73)90231-X

10. Cousins, R.R., Markham, M.F.: The use of ultrasonic spectroscopy in the location of delaminations in fibre-reinforced polymers. Composites 8(3), 145-152 (1977). https://doi.org/10.1016/0010-4361(7 7) $90008-8$

11. Fitting, D.W., Adler, L.: Applications of ultrasonic spectroscopy to materials evaluation. In: Fitting, D.W., Adler, L. (eds.) Ultrasonic Spectral Analysis for Nondestructive Evaluation, pp. 93-137. Springer, Boston (1981)

12. Wright, W.M.D., Hutchins, D.A.: Air-coupled ultrasonic testing of metals using broadband pulses in through-transmission. Ultrasonics 37(1), 19-22 (1999). https://doi.org/10.1016/S0041-624X(98 )00034-1

13. Schindel, D.W., Hutchins, D.A.: Through-thickness characterization of solids by wideband air-coupled ultrasound. Ultrasonics 33(1), 11-17 (1995). https://doi.org/10.1016/0041-624X(95)0001 $1-\mathrm{Q}$

14. Scruby, C.B., Drain, L.E.: Laser Ultrasonics: Techniques and Applications. A. Hilger, Philadelphia (1990)

15. Podymova, N.B., Karabutov, A.A.: Broadband laser-ultrasonic spectroscopy for quantitative characterization of porosity effect on acoustic attenuation and phase velocity in CFRP laminates. J. Nondestruct. Eval. 33(1), 141-151 (2014). https://doi.org/10.1007/s10 921-013-0210-z

16. Wright, W.M.D., Hutchins, D.A., Gachagan, A., Hayward, G.: Polymer composite material characterisation using a laser/airtransducer system. Ultrasonics 34(8), 825-833 (1996). https://doi. org/10.1016/S0041-624X(96)00083-2

17. Diot, G., Koudri-David, A., Walaszek, H., Guégan, S., Flifla, J.: Non-destructive testing of porosity in laser welded aluminium alloy plates: laser ultrasound and frequency-bandwidth analysis. J. Nondestruct. Eval. 32(4), 354-361 (2013). https://doi.org/10.1007/s10 921-013-0189-5

18. Fischer, B., Sarasini, F., Tirillò, J., Touchard, F., Chocinski-Arnault, L., Mellier, D., Panzer, N., Sommerhuber, R., Russo, P., Papa, I., Lopresto, V., Ecault, R.: Impact damage assessment in biocomposites by micro-CT and innovative air-coupled detection of laser-generated ultrasound. Compos. Struct. 210, 922-931 (2019). https://doi.org/10.1016/j.compstruct.2018.12.013

19. Solodov, I., Dillenz, A., Kreutzbruck, M.: A new mode of acoustic NDT via resonant air-coupled emission. J. Appl. Phys. 121(24), 245101 (2017). https://doi.org/10.1063/1.4985286

20. Solodov, I., Bai, J., Busse, G.: Resonant ultrasound spectroscopy of defects: case study of flat-bottomed holes. J. Appl. Phys. 113(22), 223512 (2013). https://doi.org/10.1063/1.4810926
21. Solodov, I., Rahammer, M., Gulnizkij, N., Kreutzbruck, M.: Noncontact sonic NDE and defect imaging via local defect resonance. J. Nondestruct. Eval. 35(3), 48 (2016). https://doi.org/10.1007/s10 921-016-0364-6

22. Segers, J., Kersemans, M., Hedayatrasa, S., Calderon, J., Van Paepegem, W.: Towards in-plane local defect resonance for nondestructive testing of polymers and composites. NDT E. Int. 98, 130-133 (2018). https://doi.org/10.1016/j.ndteint.2018.05.007

23. Hettler, J., Tabatabaeipour, M., Delrue, S., Van Den Abeele, K.: Detection and characterization of local defect resonances arising from delaminations and flat bottom holes. J. Nondestruct. Eval. 36(1), 2 (2016). https://doi.org/10.1007/s10921-016-0380-6

24. Perterer, M.: Schadensidentifikation und -bewertung von CFKBauteilen mittels phasenmodulierter Thermographie. Dissertation, Technical University of Munich (2012)

25. Fischer, B.: Optical microphone hears ultrasound. Nat Photonics 10, 356-358 (2016). https://doi.org/10.1038/nphoton.2016.95

26. Preisser, S., Rohringer, W., Liu, M., Kollmann, C., Zotter, S., Fischer, B., Drexler, W.: All-optical highly sensitive akinetic sensor for ultrasound detection and photoacoustic imaging. Biomed. Opt. Express 7(10), 4171-4186 (2016). https://doi.org/10.1364/BOE.7. 004171

27. Preisser, S., Fischer, B., Panzer, N.: Listening to ultrasound with a laser. Opt. Photon. 12(5), 22-25 (2017). https://doi.org/10.1002/ opph.201700031

28. Rus, J., Fischer, B., Grosse, C.U.: Photoacoustic inspection of CFRP using an optical microphone. Proc. SPIE Opt. Meas. Syst. Ind. Inspect. XI, 1105622 (2019). https://doi.org/10.1117/12.2525 021

29. Solodov, I., Döring, D., Busse, G.: New opportunities for NDT using non-linear interaction of elastic waves with defects. J. Mech. Eng. (2011). https://doi.org/10.1016/10.5545/sv-jme.2010.168

30. Della, C.N., Shu, D.: Vibration of delaminated composite laminates: a review. Appl. Mech. Rev. 60(1), 1-20 (2007). https://doi. org/10.1016/10.1115/1.2375141

31. Sarens, B., Verstraeten, B., Glorieux, C., Kalogiannakis, G., Hemelrijck, D.V.: Investigation of contact acoustic nonlinearity in delaminations by shearographic imaging, laser Doppler vibrometric scanning and finite difference modeling. IEEE Trans. Ultrason. Ferroelectr. Freq. Control 57(6), 1383-1395 (2010). https://doi. org/10.1109/TUFFC.2010.1557

32. Blevins, R.D.: Formulas for Natural Frequency and Mode Shape. Van Nostrand Reinhold Company, New York (1979)

Publisher's Note Springer Nature remains neutral with regard to jurisdictional claims in published maps and institutional affiliations. 